\title{
Strong curvature effects in Neumann wave problems
}

\author{
Willatzen, Morten; Pors, A.; Gravesen, Jens
}

\section{Published in:}

Journal of Mathematical Physics

Link to article, DOI:

$10.1063 / 1.4745856$

Publication date:

2012

Document Version

Publisher's PDF, also known as Version of record

Link back to DTU Orbit

Citation (APA):

Willatzen, M., Pors, A., \& Gravesen, J. (2012). Strong curvature effects in Neumann wave problems. Journal of Mathematical Physics, 53(8), 083507. https://doi.org/10.1063/1.4745856

\section{General rights}

Copyright and moral rights for the publications made accessible in the public portal are retained by the authors and/or other copyright owners and it is a condition of accessing publications that users recognise and abide by the legal requirements associated with these rights.

- Users may download and print one copy of any publication from the public portal for the purpose of private study or research.

- You may not further distribute the material or use it for any profit-making activity or commercial gain

- You may freely distribute the URL identifying the publication in the public portal

If you believe that this document breaches copyright please contact us providing details, and we will remove access to the work immediately and investigate your claim. 


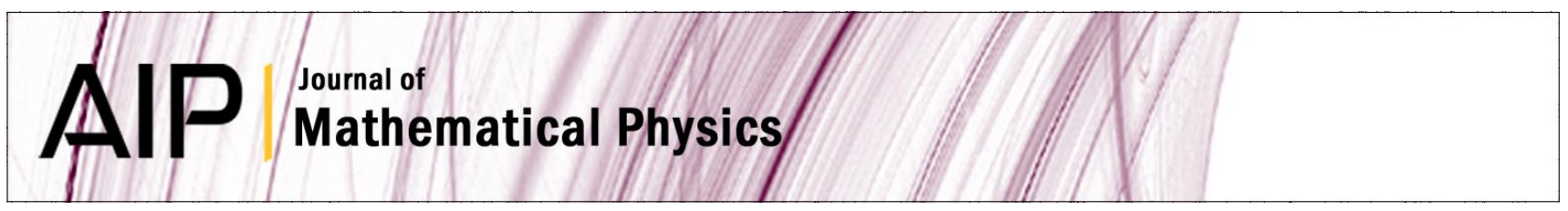

\section{Strong curvature effects in Neumann wave problems}

M. Willatzen, A. Pors, and J. Gravesen

Citation: J. Math. Phys. 53, 083507 (2012); doi: 10.1063/1.4745856

View online: http://dx.doi.org/10.1063/1.4745856

View Table of Contents: http://jmp.aip.org/resource/1/JMAPAQ/v53/i8

Published by the American Institute of Physics.

\section{Related Articles}

Optimal electromagnetic-wave absorption by enhanced dipole polarization in Ni/C nanocapsules Appl. Phys. Lett. 101, 083116 (2012)

Transmission through stacked 2D periodic distributions of square conducting patches J. Appl. Phys. 112, 033101 (2012)

New equipment for microwave electric field visualization

Rev. Sci. Instrum. 83, 074704 (2012)

Frequency-dispersive resistance of high impedance surface absorber with trapezoid-coupling pattern J. Appl. Phys. 112, 014106 (2012)

Extremely high extinction ratio terahertz broadband polarizer using bilayer subwavelength metal wire-grid structure

Appl. Phys. Lett. 101, 011101 (2012)

\section{Additional information on J. Math. Phys.}

Journal Homepage: http://jmp.aip.org/

Journal Information: http://jmp.aip.org/about/about_the_journal

Top downloads: http://jmp.aip.org/features/most_downloaded

Information for Authors: http://jmp.aip.org/authors

\section{ADVERTISEMENT}

The most comprehensive support for physics in any mathematical software package World-leading tools for performing calculations in theoretical physics

Your work in Maple matches how you would write the problems and solutions by hand

State-of-the-art environment for algebraic computations in physics

The only system with the ability to handle a wide range of physics computations as well as pencil-and-paper style input and textbook-quality display of results

- Access to Maple's full mathematical power, programming language, visualization routines, and document creation tools 


\title{
Strong curvature effects in Neumann wave problems
}

\author{
M. Willatzen, ${ }^{1, a)}$ A. Pors, ${ }^{1}$ and J. Gravesen ${ }^{2}$ \\ ${ }^{1}$ Mads Clausen Institute, University of Southern Denmark, Alsion 2, \\ DK-6400 Sønderborg, Denmark \\ ${ }^{2}$ Department of Mathematics, Technical University of Denmark, Matematiktorvet, \\ DK-2800 Kgs. Lyngby, Denmark
}

(Received 29 November 2011; accepted 30 July 2012; published online 24 August 2012)

\begin{abstract}
Waveguide phenomena play a major role in basic sciences and engineering. The Helmholtz equation is the governing equation for the electric field in electromagnetic wave propagation and the acoustic pressure in the study of pressure dynamics. The Schrödinger equation simplifies to the Helmholtz equation for a quantum-mechanical particle confined by infinite barriers relevant in semiconductor physics. With this in mind and the interest to tailor waveguides towards a desired spectrum and modal pattern structure in classical structures and nanostructures, it becomes increasingly important to understand the influence of curvature effects in waveguides. In this work, we demonstrate analytically strong curvature effects for the eigenvalue spectrum of the Helmholtz equation with Neumann boundary conditions in cases where the waveguide cross section is a circular sector. It is found that the linear-in-curvature contribution originates from parity symmetry breaking of eigenstates in circular-sector tori and hence vanishes in a torus with a complete circular cross section. The same strong curvature effect is not present in waveguides subject to Dirichlet boundary conditions where curvature contributions contribute to second-order in the curvature only. We demonstrate this finding by considering wave propagation in a circular-sector torus corresponding to Neumann and Dirichlet boundary conditions, respectively. Results for relative eigenfrequency shifts and modes are determined and compared with three-dimensional finite element method results. Good agreement is found between the present analytical method using a combination of differential geometry with perturbation theory and finite element results for a large range of curvature ratios. (C) 2012 American Institute of Physics. [http://dx.doi.org/10.1063/1.4745856]
\end{abstract}

\section{INTRODUCTION}

Waveguide phenomena play a major role in basic sciences and applications. Classical waveguide studies are limited to geometries where separable solutions can be sought in the spatial coordinates. ${ }^{1-5}$ It is known that the Helmholtz equation is separable in 11 coordinate systems. ${ }^{2,4,6}$ Among those, however, a substantial part of applications refer to the rectangular, circular cylindrical, and spherical coordinate systems.

In cases where propagation is limited to spaces where one or more dimensions are small it is possible to employ differential geometry to obtain separable solutions even in the case of complicated geometries. Four decades ago, Jensen and Koppe ${ }^{7}$ analyzed the influence of curvature on quantummechanical properties of particles confined to a two-dimensional region in three-dimensional space. This work was continued by da Costa in the early 1980s. ${ }^{8,9}$ Goldstone and Jaffe ${ }^{10}$ studied the existence of curvature-induced bound states in waveguides subject to Dirichlet boundary conditions relevant for Schrödinger problems and in electromagnetics. Further important contributions to the study of nanowire eigenvalue and eigenstate properties and bending effects can be found in Refs. 11 and 12 .

\footnotetext{
a)Electronic mail: willatzen@mci.sdu.dk.
} 


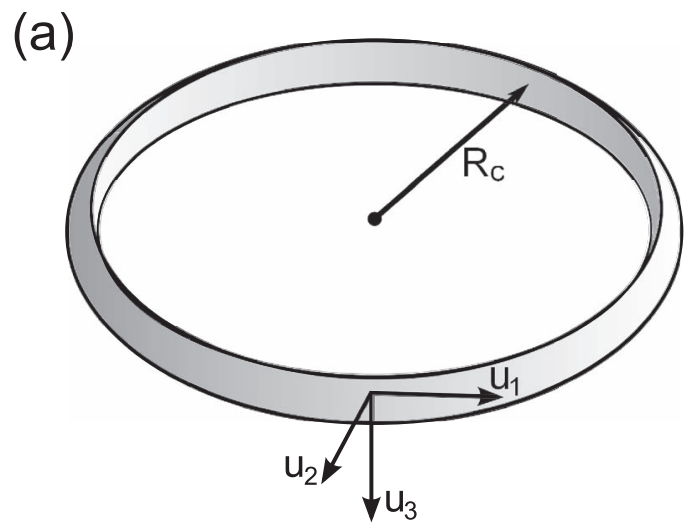

(b)

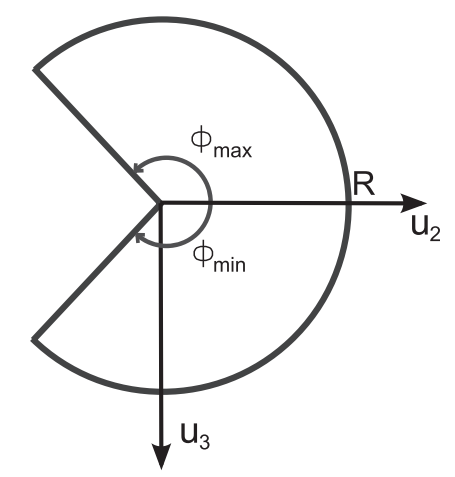

FIG. 1. Sketch of a circular-sector torus and the choice of coordinates $u_{1}, u_{2}, u_{3}$.

The possibility of growing nanostructures of almost any shape has initiated an increasing interest in tailoring structures towards prescribed physical properties such as transition energies and optical dipole moments. ${ }^{13-21}$ A similar interest exists in understanding the influence of curvature effects for electromagnetic and acoustic waveguides as well as curved cavity structures. These design studies can pave the way for discovering novel physical effects and identifying new and improved sensor applications. Examples along these lines include determination of spatial resonances and localization in metamaterials and their use in sensors. ${ }^{22-26}$

Recently, Willatzen et al. derived a formal procedure based on differential geometry and perturbation theory for identifying analytically the influence of curvature for eigenstates and eigenvalues to the Helmholtz equation subject to arbitrary boundary conditions including Dirichlet and Neumann boundary conditions ${ }^{27}$ Earlier, analytical curvature studies were restricted to the Dirichlet problem. It is shown in Ref. 27 that curvature effects give quantitatively and qualitatively different results in cases with Dirichlet and Neumann boundary conditions.

In the literature, only rather small second-order in curvature contributions to the eigenvalue spectrum of the wave equations with Dirichlet or Neumann boundary conditions have been described so far. In the present work, we identify for a circular-sector torus (see Figure 1), a linear-incurvature, and hence potentially strong, contribution to the eigenvalues for the Helmholtz equation with Neumann boundary conditions. Based on this finding, we examine linear-in-curvature frequency changes for sound wave propagation confined to a circular-sector torus.

As a corollary, we show using the present differential geometry analysis that linear-in-curvature contributions to eigenfrequencies do not exist for the circular-sector problem in the case with Dirichlet boundary conditions and that a linear-in-curvature contribution vanishes for the complete torus with circular cross section in the case with Neumann boundary conditions. Results are compared with three-dimensional finite element method analyses and good agreement is found. In particular, we demonstrate a linear dependence of eigenfrequencies on curvature using the commercial finite 
element package COMSOL in agreement with our computationally effectively differential geometry and perturbation theory method.

\section{THEORY}

Based on a formulation of the Laplacian in general curved coordinates, we recast the scalar Helmholtz equation as a differential operator problem in curved coordinates where the main (unperturbed) contribution is independent of the local structure curvature in addition to two perturbative contributions to first- and second-order, respectively, in the local radius-of-curvature. ${ }^{17}$ First, the Laplacian is derived in the so-called minimal-rotation-frame (MRF). Next, we write down general expressions for the first- and second-order perturbative contributions in the curvature. A comparison of analytic differential geometry results vs. finite element method results is carried out.

\section{A. Parametrization of a curved structure}

Consider a wire structure where the axis is given as a curve $\mathbf{x}(s)$ parametrized by arc length $s=u_{1}$. The tangent vector $\mathbf{t}(s)=\mathbf{x}^{\prime}(s)=\mathrm{d} \mathbf{x} / \mathrm{d} s$ is a unit vector field along the curve and we can augment it with vector fields $\mathbf{p}(s)$ and $\mathbf{q}(s)$ such that $\mathbf{t}(s), \mathbf{p}(s), \mathbf{q}(s)$ constitute an orthonormal frame at each point $\mathbf{x}(s)$ along the axis.

Let $U \subseteq \mathbb{R}^{3}$ be a tubular neighborhood around the $x$ axis. Consider the corresponding tubular neighborhood around the curve $\mathbf{x}(s)$ parametrized by

$$
\begin{gathered}
\tilde{\mathbf{x}}\left(s, u_{2}, u_{3}\right)=\mathbf{x}(s)+u_{2} \mathbf{p}(s)+u_{3} \mathbf{q}(s), \\
\left(s, u_{2}, u_{3}\right) \in U,
\end{gathered}
$$

where $u_{2}$ and $u_{3}$ are small numbers. We then have

$$
\begin{aligned}
\mathbf{x}_{1} & =\frac{\partial \tilde{\mathbf{x}}}{\partial s}=\mathbf{x}^{\prime}+u_{2} \mathbf{p}^{\prime}+u_{3} \mathbf{q}^{\prime} \\
& =\left(1-a u_{2}-b u_{3}\right) \mathbf{t}-c u_{3} \mathbf{p} \\
& +c u_{2} \mathbf{q}, \\
\mathbf{x}_{2} & =\frac{\partial \tilde{\mathbf{x}}}{\partial u_{2}}=\mathbf{p}, \\
\mathbf{x}_{3} & =\frac{\partial \tilde{\mathbf{x}}}{\partial u_{3}}=\mathbf{q},
\end{aligned}
$$

where

$$
a(s)=\frac{d \mathbf{t}}{d s} \cdot \mathbf{p}, b(s)=\frac{d \mathbf{t}}{d s} \cdot \mathbf{q}, c(s)=\frac{d \mathbf{p}}{d s} \cdot \mathbf{q} .
$$

The metric tensor $\left(G_{i j}=\mathbf{x}_{i} \cdot \mathbf{x}_{j}\right)$ is

$$
\left[G_{i j}\right]=\left[\begin{array}{ccc}
\left(1-a u_{2}-b u_{3}\right)^{2} & -c u_{3} & c u_{2} \\
+\left(c u_{2}\right)^{2}+\left(c u_{3}\right)^{2} & & \\
-c u_{3} & 1 & 0 \\
c u_{2} & 0 & 1
\end{array}\right] .
$$

In the following, the so-called MRF with $c=0$ is employed ${ }^{17}$ where the Laplacian takes a simple form. Further, we shall keep only terms of zeroth-order in $u_{2}$ and $u_{3}$, i.e., discarding terms of order 1 and higher [note that, e.g., $u_{3} \frac{\partial}{\partial u_{2}}$ is of order 0 since $u_{3}$ and $\frac{\partial}{\partial u_{2}}$ are of order +1 and -1 , respectively]. The Laplacian in the latter approximation, accurate for structures where the local 
radius-of-curvature is much larger than the cross-sectional radius, reads

$$
\begin{aligned}
\nabla^{2} & =G^{i j} \frac{\partial^{2}}{\partial u^{i} \partial u^{j}}+\left(G^{i j} \frac{\frac{\partial G}{\partial u^{j}}}{2 G}+\frac{\partial G^{i j}}{\partial u^{j}}\right) \frac{\partial}{\partial u^{i}} \\
& =\left(\frac{\partial^{2}}{\partial\left(u_{2}\right)^{2}}+\frac{\partial^{2}}{\partial\left(u_{3}\right)^{2}}\right)-\left(a \frac{\partial}{\partial u_{2}}+b \frac{\partial}{\partial u_{3}}\right) \\
& -\left(a^{2} u_{2} \frac{\partial}{\partial u_{2}}+b^{2} u_{3} \frac{\partial}{\partial u_{3}}+a b u_{2} \frac{\partial}{\partial u_{3}}+a b u_{3} \frac{\partial}{\partial u_{2}}\right)+\frac{\partial^{2}}{\partial s^{2}},
\end{aligned}
$$

where $G=\operatorname{det}\left(G_{i j}\right), G^{i j}=\left(G_{i j}\right)^{-1}$. This zeroth-order approximation for the Laplacian is in excellent agreement with exact results as demonstrated for electron states in a nanowire with a radius-tocurvature ratio up to $10 \% .^{16}$

\section{APPLICATION TO A TORUS OR A CIRCULAR-SECTOR TORUS}

The above formulation contains arbitrariness in the choice of $\mathbf{p}$ and $\mathbf{q}$ as a function of $u_{1}$. For a circular-sector torus, we choose the configuration shown in Figure 1 with $\mathbf{p}$ and $\mathbf{q}$ pointing along the positive $u_{2}$ and $u_{3}$ directions, respectively. This choice leads to $a=-\frac{1}{R_{c}}$ and $b=0$ with $R_{c}$ the major torus radius measured from the center of the torus to the center of the torus cross section. Note that the negative sign for $a$ is due to $u_{2}$ increasing away from the torus center, i.e., $\frac{d \mathbf{t}}{d s} \times \mathbf{p}<0$. An arc-length parametrization for the torus or, equivalently, the circular-sector torus centerline is

$$
\mathbf{r}\left(u^{1}\right)=\left(R_{c} \cos \left(\frac{u^{1}}{R_{c}}\right), R_{c} \sin \left(\frac{u^{1}}{R_{c}}\right), 0\right),
$$

where $0 \leq u_{1} \leq 2 \pi R_{c}$.

\section{A. Wave propagation problems}

Consider the wave equation problem

$$
\nabla^{2} \psi+\frac{\omega^{2}}{c^{2}} \psi=0
$$

where $\psi$ is the eigensolution, $\frac{\omega}{2 \pi}$ is the wave frequency, and $c$ is the wave speed.

Boundary conditions are often of Neumann or Dirichlet type. For example, the boundary condition for sound waves confined to a circular-sector torus waveguide with rigid walls is a Neumann problem

$$
\frac{\partial \psi}{\partial n}=0
$$

where $n$ denotes the boundary normal. In contrast, the boundary condition for electrons confined by infinite barriers in, e.g., the effective-mass approximation used in solid state physics is a Dirichlet problem

$$
\psi=0
$$

In the effective-mass approximation, $\frac{\omega^{2}}{c^{2}}$ in Eq. (9) is replaced by $\frac{2 m_{e f f} E}{\hbar^{2}}$ where $m_{e f f}, E$, and $\hbar$ are the electron effective mass, the electron energy, and Planck's constant divided by $2 \pi$, respectively.

Using Eq. (7), $a=-\frac{1}{R_{c}}, b=0$, and the fact that the centerline parametrization is an arc-length parametrization: $u_{1}=s$, Eq. (9) can be expressed as the operator eigenvalue problem

$$
\mathcal{L} \psi \equiv \mathcal{L}_{0} \psi+\mathcal{L}_{1} \psi=-\frac{\omega^{2}}{c^{2}} \psi
$$




$$
\begin{aligned}
& \mathcal{L}_{0}=\frac{\partial^{2}}{\partial\left(u_{1}\right)^{2}}+\frac{\partial^{2}}{\partial\left(u_{2}\right)^{2}}+\frac{\partial^{2}}{\partial\left(u_{3}\right)^{2}}, \\
& \mathcal{L}_{1}=\frac{1}{R_{c}} \frac{\partial}{\partial u_{2}}-\frac{1}{R_{c}^{2}} u_{2} \frac{\partial}{\partial u_{2}}
\end{aligned}
$$

where $\mathcal{L}_{0}$ and $\mathcal{L}_{1}$ are operator contributions to zeroth-order and up to second-order in the curvature $\frac{1}{R_{c}}$, respectively. This is a standard eigenvalue problem for determination of mode frequencies $f_{n}=\frac{\omega_{n}}{2 \pi}$ and eigenfunctions $\psi_{n}$ where $n$ designates the mode number.

Note that neither the circular-sector torus nor the torus can be handled as a separable problem. Our present differential-geometry formulation allows a quasi-analytical solution to the (circularsector) torus problem using perturbation theory exact to second-order in the curvature $\frac{1}{R_{c}}$.

\section{FIRST-ORDER CURVATURE CONTRIBUTIONS}

In this section, we derive first-order curvature contributions to eigenfrequency shifts for a circular-sector torus and the torus in the case of Neumann and Dirichlet boundary conditions.

\section{A. Neumann boundary conditions - Circular-sector torus}

Normalized eigenstates to the unperturbed problem satisfying Neumann boundary conditions at all boundary sections are

$$
\begin{aligned}
|l m n\rangle & =N_{l m n} g_{l}\left(u_{1}\right) J_{m}\left(k_{m n} \rho\right) \cos \left(m \phi+\alpha_{m}\right), \\
m & =\frac{p \pi}{\phi_{\max }-\phi_{\min }}, \\
\alpha_{m} & =-m \phi_{\min }, \\
k_{m n} J_{m}^{\prime}\left(k_{m n} R\right) & =0, \\
\frac{1}{N_{l m n}^{2}} & =\int_{R_{c} \phi_{\min }}^{R_{c} \phi_{\max }} g_{l}^{2}\left(u_{1}\right) d u_{1} \int_{0}^{R} J_{m}^{2}\left(k_{m n} \rho\right) \rho d \rho \int_{\phi_{\min }}^{\phi_{\max }} \cos ^{2}\left(m \phi+\alpha_{m}\right) d \phi,
\end{aligned}
$$

where $\rho$ and $\phi$ are polar coordinates in the structure cross section, $p$ is an integer (including 0 ), and $R$ is the radius of the circular-sector torus cross section. Consider in the following $g_{l}$ to fulfill

$$
\int_{R_{c} \phi_{\min }}^{R_{c} \phi_{\max }} g_{l}^{2}\left(u_{1}\right) d u_{1}=1
$$

Since

$$
\begin{aligned}
u_{2} & =\rho \cos \phi, \\
\frac{\partial}{\partial u_{2}} & =\cos \phi \frac{\partial}{\partial \rho}-\frac{\sin \phi}{\rho} \frac{\partial}{\partial \phi},
\end{aligned}
$$

the first-order perturbative contribution in $\frac{1}{R_{c}}$ is

$$
\begin{aligned}
\left\langle l m n\left|\frac{1}{R_{c}} \frac{\partial}{\partial u_{2}}\right| l m n\right\rangle & =\frac{1}{R_{c}} N_{l m n}^{2}\left[\int_{0}^{R} \rho J_{m}\left(k_{m n} \rho\right) \frac{d}{d \rho} J_{m}\left(k_{m n} \rho\right) d \rho \int_{\phi_{\min }}^{\phi_{\max }} \cos ^{2}\left(m \phi+\alpha_{m}\right) \cos \phi d \phi\right. \\
& \left.+m \int_{0}^{R} J_{m}^{2}\left(k_{m n} \rho\right) d \rho \int_{\phi_{\min }}^{\phi_{\max }} \cos \left(m \phi+\alpha_{m}\right) \sin \left(m \phi+\alpha_{m}\right) \sin \phi d \phi\right]
\end{aligned}
$$


Further,

$$
\begin{aligned}
& \int_{0}^{R} \rho J_{m}\left(k_{m n} \rho\right) \frac{d}{d \rho} J_{m}\left(k_{m n} \rho\right) d \rho=\frac{1}{2} \int_{0}^{R} \rho \frac{d}{d \rho}\left[J_{m}^{2}\left(k_{m n} \rho\right)\right] d \rho=\frac{1}{2} R J_{m}^{2}\left(k_{m n} R\right) \\
& -\frac{1}{2} \int_{0}^{R} J_{m}^{2}\left(k_{m n} \rho\right) d \rho \\
& \int_{\phi_{\min }}^{\phi_{\max }} \cos ^{2}\left(m \phi+\alpha_{m}\right) \cos \phi d \phi=\left[\frac{1}{2} \sin \phi+\frac{1}{4(2 m-1)} \sin \left((2 m-1) \phi+2 \alpha_{m}\right)\right. \\
& \left.+\frac{1}{4(2 m+1)} \sin \left((2 m+1) \phi+2 \alpha_{m}\right)\right]_{\phi_{\min }}^{\phi_{\max }} \text {, } \\
& \int_{\phi_{\min }}^{\phi_{\max }} \cos \left(m \phi+\alpha_{m}\right) \sin \left(m \phi+\alpha_{m}\right) \sin \phi d \phi=\left[\frac{1}{4(2 m-1)} \sin \left((2 m-1) \phi+2 \alpha_{m}\right)\right. \\
& \left.-\frac{1}{4(2 m+1)} \sin \left((2 m+1) \phi+2 \alpha_{m}\right)\right]_{\phi_{\min }}^{\phi_{\max }} \\
& \frac{1}{N_{l m n}^{2}}=\frac{R^{2}}{2}\left(1-\frac{m^{2}}{k_{m n}^{2} R^{2}}\right) J_{m}^{2}\left(k_{m n} R\right)\left[\frac{\phi}{2}+\frac{1}{4 m} \sin \left(2 m \phi+2 \alpha_{m}\right)\right]_{\phi_{\min }}^{\phi_{\max }} .
\end{aligned}
$$

For the circular-sector torus, the normalized eigenstates $g_{l}$, periodic in $2 \pi R_{c}$, are

$$
g_{l}\left(u_{1}\right)=\frac{1}{\sqrt{\pi\left(1+\delta_{l 0}\right) R_{c}}} \sin \left(\frac{l}{R_{c}} u_{1}+v\right),
$$

where $l$ is an integer, and $v$ is an arbitrary phase. The unperturbed eigenfrequencies become

$$
f_{l m n, 0}^{N}=\frac{\omega_{l m n, 0}}{2 \pi}=\frac{c}{2 \pi} \sqrt{k_{m n}^{2}+\frac{l^{2}}{R_{c}^{2}}} .
$$

The change in eigenfrequencies stemming from first-order contributions in curvature is

$$
\Delta\left(f_{l m n}^{N}\right)^{2}=\frac{\Delta \omega_{l m n}^{2}}{(2 \pi)^{2}}=-\frac{c^{2}}{(2 \pi)^{2}}\left\langle l m n\left|\frac{1}{R_{c}} \frac{\partial}{\partial u_{2}}\right| l m n\right\rangle,
$$

with superscript $N$ denoting Neumann boundary conditions, and the relative change reads

$$
\frac{\Delta\left(f_{l m n}^{N}\right)^{2}}{\left(f_{l m n, 0}^{N}\right)^{2}}=-\frac{\frac{c^{2}}{(2 \pi)^{2}}\left\langle l m n\left|\frac{1}{R_{c}} \frac{\partial}{\partial u_{2}}\right| l m n\right\rangle}{\left(f_{l m n, 0}^{N}\right)^{2}}=-\frac{\left\langle l m n\left|\frac{1}{R_{c}} \frac{\partial}{\partial u_{2}}\right| l m n\right\rangle}{k_{m n}^{2}+\frac{l^{2}}{R_{c}^{2}}},
$$

or

$$
\frac{\Delta f_{l m n}^{N}}{f_{l m n, 0}^{N}}=-\frac{1}{2} \frac{\left\langle l m n\left|\frac{1}{R_{c}} \frac{\partial}{\partial u_{2}}\right| l m n\right\rangle}{k_{m n}^{2}+\frac{l^{2}}{R_{c}^{2}}} .
$$

Thus, the above calculation of the matrix element in Eq. (23) concludes the derivation of first-order curvature changes to $f_{l m n}^{N}$. Our differential geometry and perturbation theory analysis shows that this matrix element is nonzero in the case of Neumann boundary conditions (refer to Sec. VI) and hence eigenfrequency changes due to curvature scale to first-order in curvature which is a new result. Earlier curvature analyses ${ }^{7-10,16-19,27}$ only demonstrated curvature effects to energies (frequencies) being of second-order in the curvature. The present finding of first-order curvature contributions is expected to dominate above second-order curvature contributions in a large range of curvatures as our FEM results confirm (refer to Sec. VI). 


\section{B. Neumann boundary conditions - Torus}

For a torus, where the cross section is a complete circle, the unperturbed eigenstates with Neumann boundary conditions are

$$
\begin{aligned}
& |l m n\rangle=N_{l m n} g_{l}\left(u_{1}\right) J_{m}\left(k_{m n} \rho\right) \sin (m \phi), \\
& |l m n\rangle=N_{l m n} g_{l}\left(u_{1}\right) J_{m}\left(k_{m n} \rho\right) \cos (m \phi),
\end{aligned}
$$

where $m$ is an integer (including $m=0$ for the $\cos (m \phi)$ solution). For the torus, since all eigenstates are parity eigenstates (with eigenvalue \pm 1 ) and $\frac{\partial}{\partial u_{2}} \rightarrow-\frac{\partial}{\partial u_{2}}$ subject to the transformation $\phi \rightarrow \phi$ $+\pi$, we immediately conclude that

$$
\frac{\Delta f_{l m n}^{N}}{f_{l m n, 0}^{N}}=0
$$

Hence, for the complete Neumann torus problem there is no first-order curvature contribution to the frequency!

\section{Dirichlet boundary conditions - Circular-sector torus}

Next, we consider Dirichlet boundary conditions at all boundary sections. In this case, normalized eigenstates to the unperturbed problem are

$$
\begin{aligned}
|l m n\rangle & =N_{l m n} g_{l}\left(u_{1}\right) J_{m}\left(k_{m n} \rho\right) \sin \left(m \phi+\alpha_{m}\right), \\
m & =\frac{p \pi}{\phi_{\max }-\phi_{\min }}, \\
\alpha_{m} & =-m \phi_{\min }, \\
J_{m}\left(k_{m n} R\right) & =0, \\
\frac{1}{N_{l m n}^{2}} & =\int_{R_{c} \phi_{\min }}^{R_{c} \phi_{\max }} g_{l}^{2}\left(u_{1}\right) d u_{1} \int_{0}^{R} J_{m}^{2}\left(k_{m n} \rho\right) \rho d \rho \int_{\phi_{\min }}^{\phi_{\max }} \sin ^{2}\left(m \phi+\alpha_{m}\right) d \phi,
\end{aligned}
$$

where $p$ is a positive integer and the first-order perturbative contribution in $\frac{1}{R_{c}}$ is

$$
\begin{aligned}
\left\langle l m n\left|\frac{1}{R_{c}} \frac{\partial}{\partial u_{2}}\right| l m n\right\rangle & =\frac{1}{R_{c}} N_{l m n}^{2}\left[\int_{0}^{R} \rho J_{m}\left(k_{m n} \rho\right) \frac{d}{d \rho} J_{m}\left(k_{m n} \rho\right) d \rho \int_{\phi_{\min }}^{\phi_{\max }} \sin ^{2}\left(m \phi+\alpha_{m}\right) \cos \phi d \phi\right. \\
& \left.-m \int_{0}^{R} J_{m}^{2}\left(k_{m n} \rho\right) d \rho \int_{\phi_{\min }}^{\phi_{\max }} \cos \left(m \phi+\alpha_{m}\right) \sin \left(m \phi+\alpha_{m}\right) \sin \phi d \phi\right] .
\end{aligned}
$$

The relevant integrals are

$$
\begin{aligned}
\int_{0}^{R} \rho J_{m}\left(k_{m n} \rho\right) \frac{d}{d \rho} J_{m}\left(k_{m n} \rho\right) d \rho & =-\frac{1}{2} \int_{0}^{R} J_{m}^{2}\left(k_{m n} \rho\right) d \rho \\
\int_{\phi_{\min }}^{\phi_{\max }} \sin ^{2}\left(m \phi+\alpha_{m}\right) \cos \phi d \phi & =\left[\frac{1}{2} \sin \phi-\frac{1}{4(2 m-1)} \sin \left((2 m-1) \phi+2 \alpha_{m}\right)\right. \\
& \left.-\frac{1}{4(2 m+1)} \sin \left((2 m+1) \phi+2 \alpha_{m}\right)\right]_{\phi_{\min }}^{\phi_{\max }}, \\
\int_{\phi_{\min }}^{\phi_{\max }} \cos \left(m \phi+\alpha_{m}\right) \sin \left(m \phi+\alpha_{m}\right) \sin \phi d \phi & =\left[\frac{1}{4(2 m-1)} \sin \left((2 m-1) \phi+2 \alpha_{m}\right)\right. \\
& \left.-\frac{1}{4(2 m+1)} \sin \left((2 m+1) \phi+2 \alpha_{m}\right)\right]_{\phi_{\min }}^{\phi_{\max }}
\end{aligned}
$$


Following steps similar to those in Subsection IV A (with superscript $D$ denoting Dirichlet boundary conditions)

$$
f_{l m n, 0}^{D}=\frac{\omega_{l m n, 0}}{2 \pi}=\frac{c}{2 \pi} \sqrt{k_{m n}^{2}+\frac{l^{2}}{R_{c}^{2}}},
$$

and the relative change in eigenfrequencies due to curvature reads

$$
\frac{\Delta\left(f_{l m n}^{D}\right)^{2}}{\left(f_{l m n, 0}^{D}\right)^{2}}=-\frac{\frac{c^{2}}{(2 \pi)^{2}}\left\langle l m n\left|\frac{1}{R_{c}} \frac{\partial}{\partial u_{2}}\right| l m n\right\rangle}{\left(f_{l m n, 0}^{D}\right)^{2}}=-\frac{\left\langle l m n\left|\frac{1}{R_{c}} \frac{\partial}{\partial u_{2}}\right| l m n\right\rangle}{k_{m n}^{2}+\frac{l^{2}}{R_{c}^{2}}} .
$$

An exact evaluation using Eqs. (40)-(43) yields

$$
\frac{\Delta f_{l m n}^{D}}{f_{l m n, 0}^{D}}=0 .
$$

Hence, for the Dirichlet circular-sector torus problem there is no first-order contribution in curvature to the frequency.

\section{Dirichlet boundary conditions - Torus}

For a torus, the unperturbed eigenstates with Dirichlet boundary conditions are

$$
\begin{aligned}
|l m n\rangle & =N_{l m n} g_{l}\left(u_{1}\right) J_{m}\left(k_{m n} \rho\right) \sin (m \phi), \\
|l m n\rangle & =N_{l m n} g_{l}\left(u_{1}\right) J_{m}\left(k_{m n} \rho\right) \cos (m \phi),
\end{aligned}
$$

where $m$ is an integer (including $m=0$ for the $\cos (m \phi)$ solution). As in the case with Neumann boundary conditions, since all eigenstates for the torus are parity eigenstates (with eigenvalue \pm 1 ) and $\frac{\partial}{\partial u_{2}} \rightarrow-\frac{\partial}{\partial u_{2}}$ subject to the transformation $\phi \rightarrow \phi+\pi$ we conclude that

$$
\frac{\Delta f_{l m n}^{D}}{f_{l m n, 0}^{D}}=0
$$

Hence, for the Dirichlet torus problem there is no first-order curvature contribution to the frequency.

\section{SECOND-ORDER CURVATURE CONTRIBUTIONS}

In addition to the first-order contributions in curvature derived above, there are frequency contributions to second-order in curvature. These arise in first-order perturbation theory due to the term $-\frac{1}{R_{c}^{2}} u_{2} \frac{\partial}{\partial u_{2}}$ and second-order perturbation theory from the term $\frac{1}{R_{c}} \frac{\partial}{\partial u_{2}}$, refer to Eq. (14). The combined second-order curvature contribution is

$$
\Delta\left(f_{l m n}\right)^{2}=-\frac{c^{2}}{(2 \pi)^{2}}\left\langle l m n\left|-\frac{1}{R_{c}^{2}} u_{2} \frac{\partial}{\partial u^{2}}\right| l m n\right\rangle-\frac{c^{2}}{(2 \pi)^{2}} \sum_{\left|l^{\prime} m^{\prime} n^{\prime}\right\rangle} \frac{\left\langle l m n\left|\frac{1}{R_{c}} \frac{\partial}{\partial u_{2}}\right| l^{\prime} m^{\prime} n^{\prime}\right\rangle\left\langle l^{\prime} m^{\prime} n^{\prime}\left|\frac{1}{R_{c}} \frac{\partial}{\partial u_{2}}\right| l m n\right\rangle}{\left(-k_{m n}^{2}-\frac{l^{2}}{R_{c}^{2}}\right)-\left(-k_{m^{\prime} n^{\prime}}^{2}-\frac{l^{2}}{R_{c}^{2}}\right)} .
$$

The second-order curvature contribution is generally non-vanishing for both Dirichlet and Neumann boundary conditions as well as for the circular-sector torus and the torus. Evidently for small curvatures, as is usually the case, the second-order curvature contribution to the frequency change is less important as compared to the first-order curvature contribution assuming the latter is allowed by symmetry. We emphasize that a simple analytical expression for the second-order curvature contribution does not exist. 


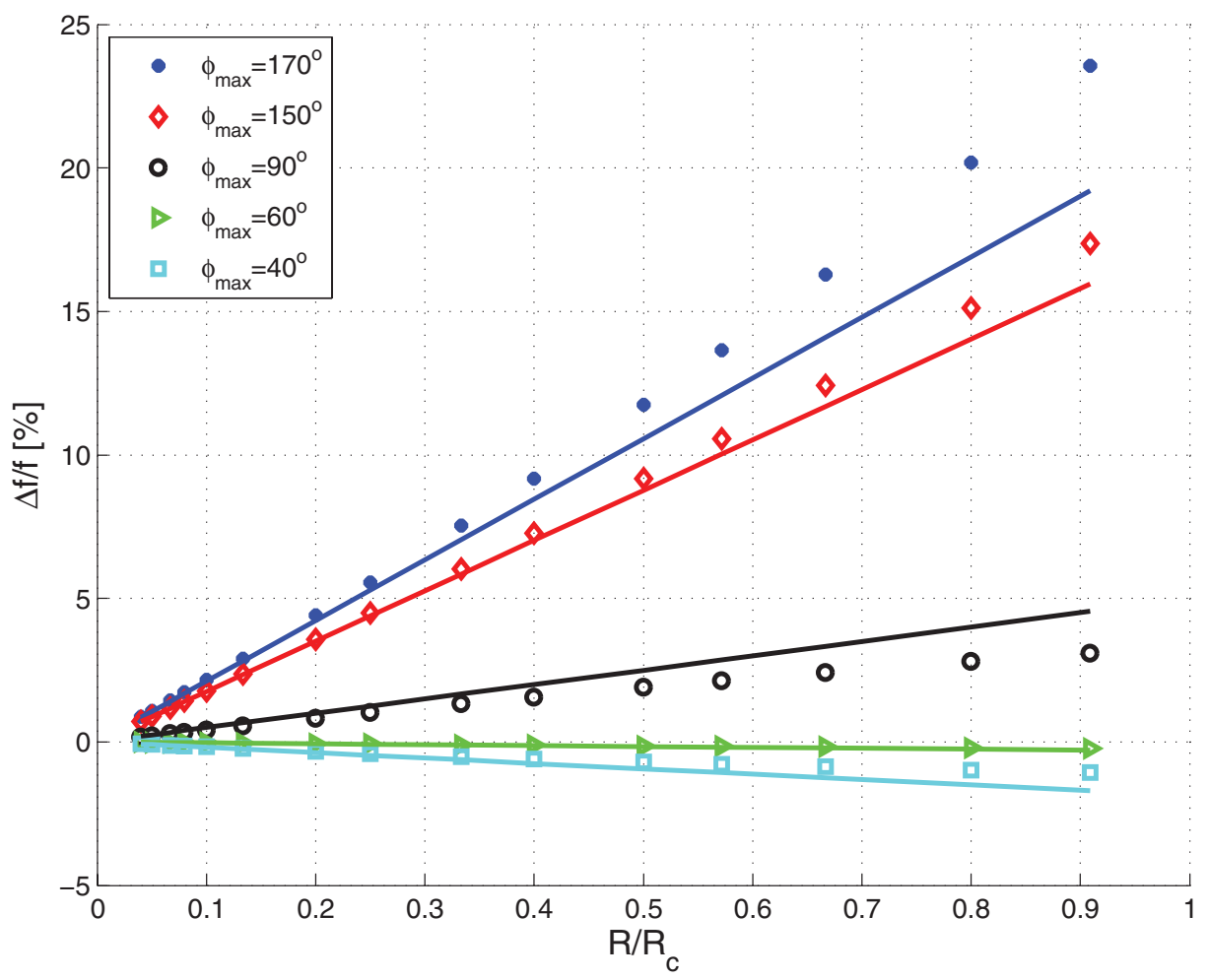

FIG. 2. First-order contribution to $\frac{\Delta f_{l m n}^{N}}{f_{l m n, 0}^{N}}$ as a function of the ratio $R / R_{c}$ corresponding to the mode $p=1, l=0, n=1$ for different angles $\phi_{\max }$. Solid line data are computed using the DGPT method, while discrete symbol points (asterisks, diamond, etc.) are found using the FEM. In the calculations, it is assumed that $\phi_{\min }=-\phi_{\max }$.

\section{RESULTS AND DISCUSSIONS}

In this section, we assume Neumann boundary conditions in accordance with the result that the first-order curvature contribution is identically zero in the case of Dirichlet boundary conditions (refer to Sec. II).

First, note that a simple expression for the integral

$$
\int_{0}^{R} J_{m}^{2}\left(k_{m n} \rho\right) d \rho
$$

does not exist and a simple expression for the frequency shift is not possible [refer to Eqs. (24) and (41)]. Instead, in Figures 2 and 3, we plot the relative frequency change, evaluated numerically based on Eq. (23), for the first mode with $p=1, l=0, n=1$. Figure 2 shows $\Delta f / f$ as a function of the ratio $R / R_{c}$ corresponding to different angles $\phi_{\max }$ and assuming $\phi_{\min }=-\phi_{\max }$. Solid lines are data computed using the present theory based on differential geometry and first-order theory (DGPT), while discrete symbol points are found using the finite element method. As shown in Sec. II, there is a non-vanishing first-order contribution in the curvature. This contribution dominates for $R / R_{c}$ ratios below approximately 0.3 . For $R / R_{c}$ values above 0.3 a nonlinear dependence becomes apparent reflecting the importance of second-order (and higher order) terms in $R / R_{c}$. Another interesting conclusion is that for smaller $\phi_{\max }$ values frequency shifts due to curvature effects are negative and rather small, while for $\phi_{\max }$ values approaching $170^{\circ}$ the frequency shift can become several percents even at $R / R_{c}$ values down to 0.1 . Furthermore, good agreement is demonstrated between DGPT and FEM for all angles $\phi_{\max }$ as long as $R / R_{c}$ is less than about 0.3 in accordance with the assumption made in first-order perturbation theory. Needless to say but the computational costs in carrying out the finite element calculations are orders of magnitude larger than for the DGPT method. 


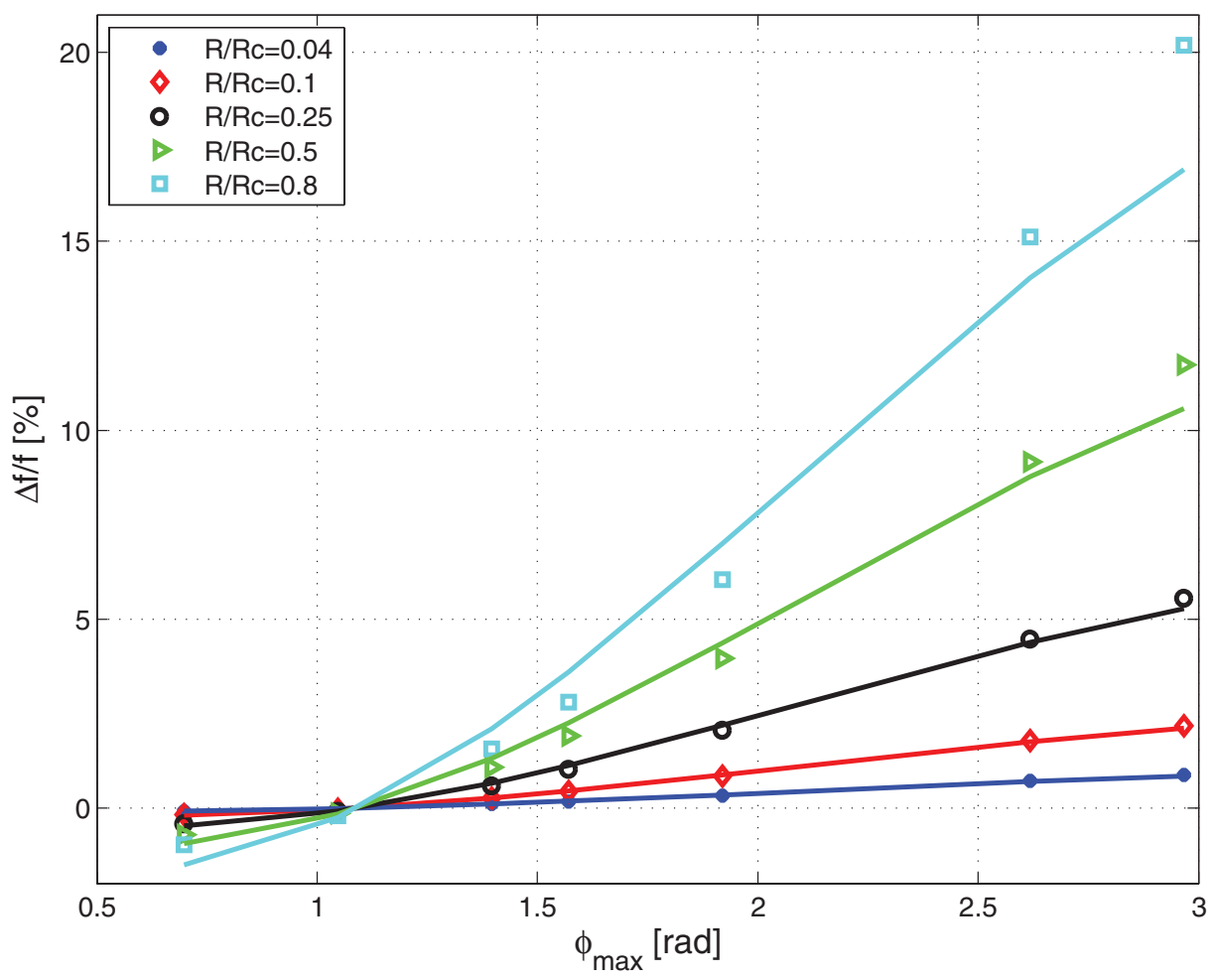

FIG. 3. First-order contribution to $\frac{\Delta f_{l n}^{N}}{f_{l m n, 0}^{N}}$ as a function of $\phi_{\max }$ corresponding to the mode $p=1, l=0, n=1$ for different ratios $R / R_{c}$. Solid line data are computed using the DGPT method, while discrete symbol points (asterisks, diamond, etc.) are found using the FEM. In the calculations, it is assumed that $\phi_{\min }=-\phi_{\max }$.

In the COMSOL computations, we used the Graphical User Interface (GUI)-based Partial Differential Equation (PDE) interface to setup the Helmholtz eigenvalue problem [Eq. (9)] and the geometry builder to create the circular-sector torus (Figure 1). The Neumann boundary condition [Eq. (10)] was applied on all faces of the torus in order to mimic hard wall boundaries. One should note that we only consider curvature effects on eigenfunctions with $l=0$, i.e., eigenfunctions that are constant with respect to $u_{1}$. For this reason larger FEM $R / R_{c}$ value data were obtained by considering numerically a smaller $u_{1}$ range than $0 \leq u_{1} \leq 2 \pi R_{c}$, and by invoking Neumann boundary conditions (i.e., periodic boundary condition) at the $u_{1}$ boundaries, hereby allowing for a constant solution along the $u_{1}$ coordinate. The advantage of only considering a fraction of the circular-sector torus is a considerable reduction in the number of mesh elements. For all FEM calculations, we used a tetrahedral mesh, and for each geometry a series of calculations with increasing mesh density were performed to ensure convergence of the found eigenfrequencies. It was found that tori with sharp-pointed cross sections, like for $\phi_{\max }=170^{\circ}$, are especially computationally demanding due to the need of a high-density mesh around $\left(u_{2}, u_{3}\right)=(0,0)$ [Figure 1(b)].

In Figure 3, relative frequency changes are plotted vs. $\phi_{\max }$ for the fundamental mode with $p=1, l=0, n=1$ in cases with different curvature ratios $R / R_{c}$. Clearly for small curvature ratios, the agreement between the present DGPT method and the FEM method is good as long as moderate curvature ratios are considered, i.e., for $R / R_{c}$ below 0.25 . The deviation between DGPT and FEM results for larger curvature ratios $\left(0.5\right.$ or higher) at all angles $\phi_{\max }$ reflects the importance of second-order perturbative terms in the derivation of frequency shifts.

It is clear from Figures 2 and 3 that the largest curvature contributions for the fundamental mode exist for $\phi_{\max }$ close to $180^{\circ}$ independent of the ratio $R / R_{c}$. This observation is, however, not easily realized with the DGPT approach due to the lack of simple explicit formulas. Therefore, it might help the understanding to study the eigenfunctions of the unperturbed cylinder cavity $|\operatorname{lmn}\rangle$ 

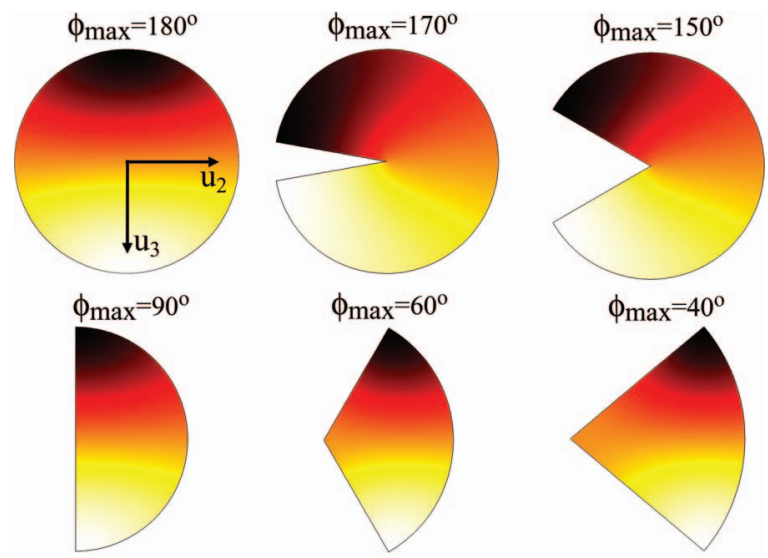

FIG. 4. Cross section of the fundamental mode $(p=1, l=0, n=1)$ in the unperturbed cylinder cavity for six different values of $\phi_{\max }$. In the calculations, it is assumed that $\phi_{\min }=-\phi_{\max }$. The mode profiles and the color scale are symmetric.

(Figure 4) since the eigenfrequency change depends on the element $\left\langle\operatorname{lmn}\left|\frac{1}{R_{c}} \frac{\partial}{\partial u_{2}}\right| l m n\right\rangle$. It is thus clear that the largest change in frequency occurs for the cross section with the (on average) most asymmetric mode profile and largest variation along the $u_{2}$ direction. From Figure 4 , and in agreement with previous reasoning, it is evident that the first-order curvature contribution for $\phi_{\max }=180^{\circ}$ (circular cross section) must be zero since the mode is symmetric along $u_{2}$. Similarly, it is seen that $\phi_{\max }$-values close to $180^{\circ}$ (see, e.g., $\phi_{\max }=170^{\circ}$ ) create mode profiles that are asymmetric and with large variation along $u_{2}$, thereby showing the highest sensitivity to the curvature. It also follows from

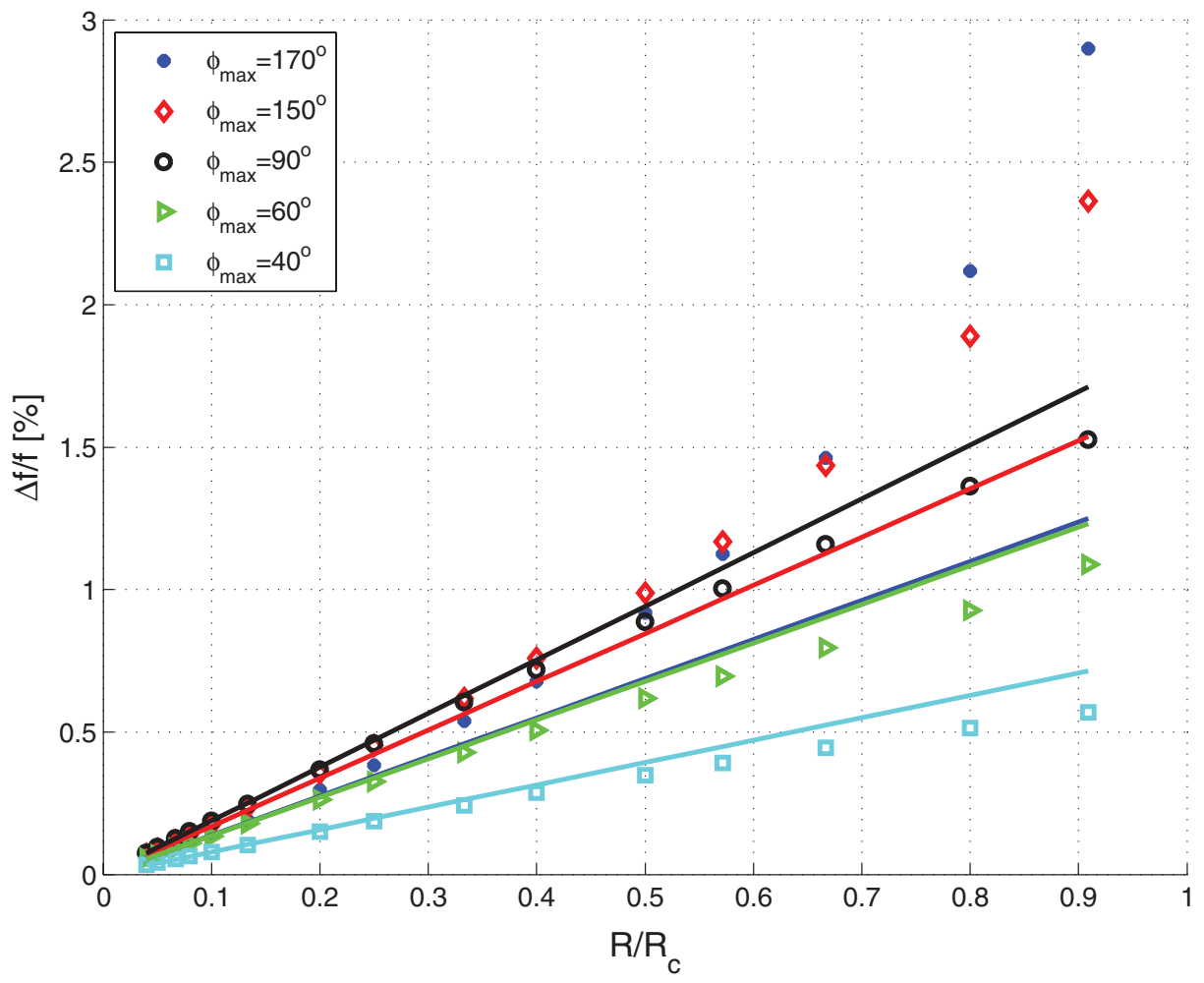

FIG. 5. First-order contribution to $\frac{\Delta f_{l m n}^{N}}{f_{l m n, 0}^{N}}$ as a function of the ratio $R / R_{c}$ corresponding to the mode $p=1, l=0, n=2$ for different angles $\phi_{\max }$. Solid line data are computed using the DGPT method, while discrete symbol points (asterisks, diamond, etc.) are found using the FEM. In the calculations, it is assumed that $\phi_{\min }=-\phi_{\max }$. 


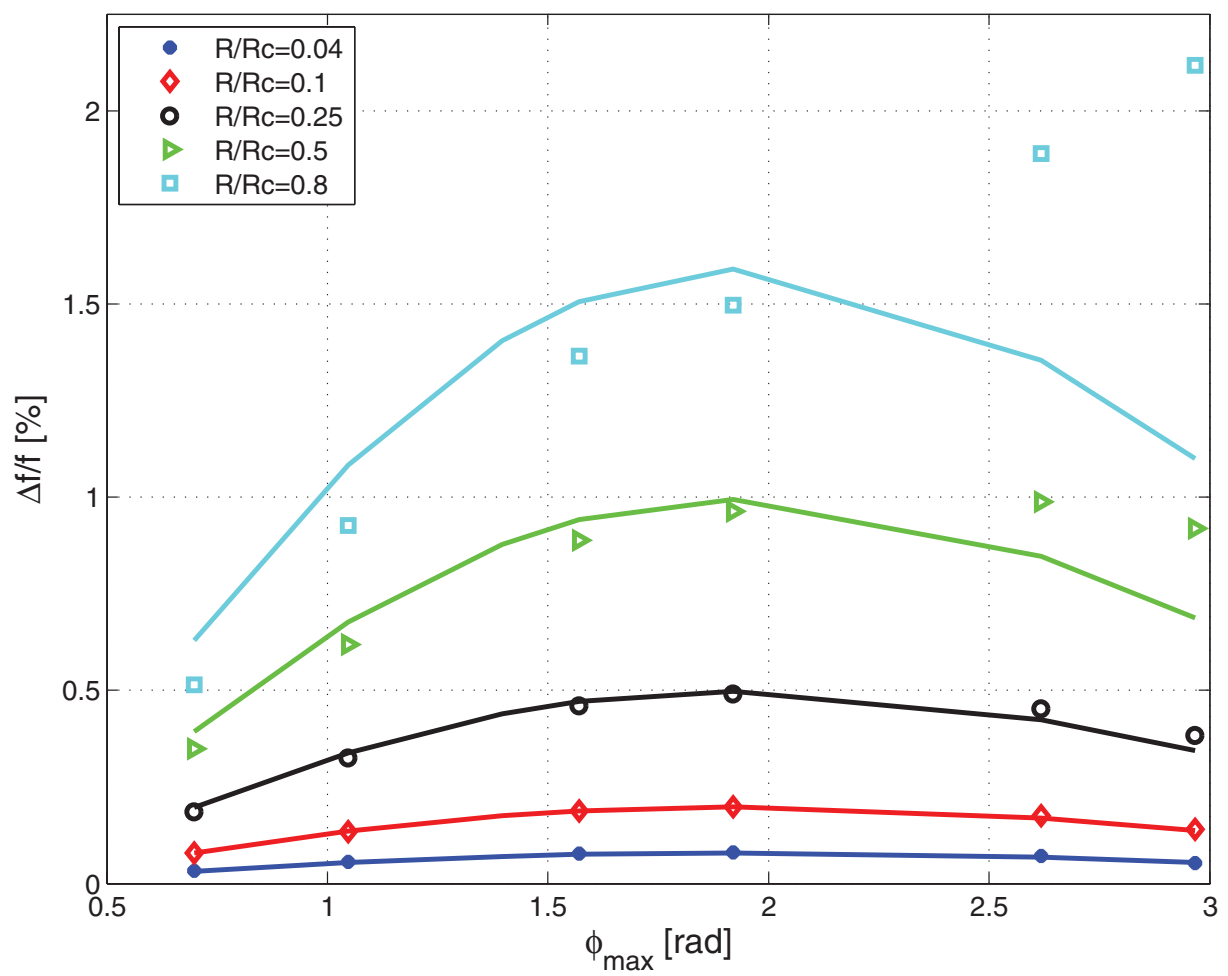

FIG. 6. First-order contribution to $\frac{\Delta f_{l m n}^{N}}{f_{l m n, 0}^{N}}$ as a function of $\phi_{\max }$ corresponding to the mode $p=1, l=0, n=2$ for different ratios $R / R_{c}$. Solid line data are computed using the DGPT method, while discrete symbol points (asterisks, diamond, etc.) are found using the FEM. In the calculations, it is assumed that $\phi_{\min }=-\phi_{\max }$.

the mode profiles that for $\phi_{\max }=60^{\circ}$ and $\phi_{\max }=40^{\circ}$ the curvature effect is small since the modes show reduced asymmetry and small variation along $u_{2}$.

In Figure 5, we consider the first excited mode along the radial direction, i.e., the mode with indices $p=1, l=0, n=2$. Results for $\Delta f l f$ plotted vs. $R / R_{c}$ for different $\phi_{\max }$ values again verify good agreement between DGPT and FEM model results for all angles $\phi_{\max }$ if $R / R_{c}$ is below approximately 0.3. In Figure 6, we observe that relative frequency shifts for the mode $p=1, l=0, n=2$ have a maximum as a function of $\phi_{\max }$ near 1.9 radians equivalent to $109^{\circ}$. This is a different conclusion as compared to the case of the fundamental mode $p=1, l=0, n=1$ where relative frequency changes continue to increase as the curvature ratio $R / R_{c}$ increases.

As an aid in understanding Figures 5 and 6, we show in Figure 7 the mode profiles for the unperturbed eigenfunction for six different values of $\phi_{\max }$. It is evident that $\phi_{\max }<180^{\circ}$ will break the symmetry of the mode along $u_{2}$, thereby creating first-order curvature contributions. At the same time, however, it is clear that the node introduced in the radial direction will typically create both positive and negative mode profile slopes along $u_{2}$ and reduce the curvature effect upon integration. This explains why the curvature effect is approximately one order of magnitude smaller compared to the fundamental mode ( $p=1, l=0, n=1)$. It is not possible with pure visualization to explain why the maximum curvature effect occurs at exactly $\phi_{\max }=109^{\circ}$ (hence, the reason why the mode profile is not shown in Figure 7). However, by visual inspection of the mode profiles it can be seen that for both large and small $\phi_{\max }$ (e.g., $\phi_{\max }=170^{\circ}$ and $\phi_{\max }=40^{\circ}$ ) the maxima and minima in the mode profiles bend towards the $u_{2}$ axis thus reducing the integral $\left\langle\operatorname{lmn}\left|\frac{1}{R_{c}} \frac{\partial}{\partial u_{2}}\right| \operatorname{lm} n\right\rangle$. Consequently, it is expected that the largest first-order curvature effect must occur between the two extrema of $\phi_{\max }$.

We next consider circular-sector tori defined by the angular interval $\phi_{\min }=-\pi-\phi_{\max }$ with $\phi_{\max }$ in the range $[-\pi / 2 ; \pi / 2]$. In Figure 8, discrete data points for the mode $p=1, l=0, n=1$ relative frequency shifts are calculated as a function of $R / R_{c}$ for different $\phi_{\max }$ values using the finite 

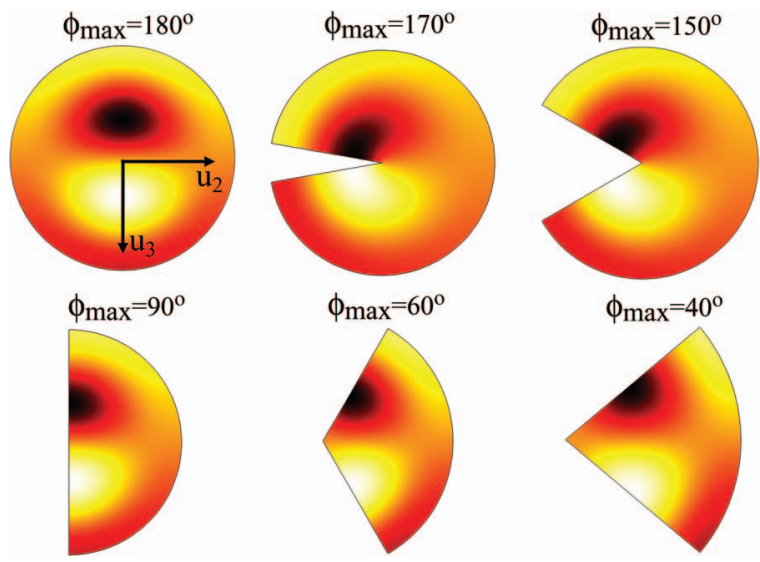

FIG. 7. Cross section of the first excited mode along the radial direction $(p=1, l=0, n=2)$ in the unperturbed cylinder cavity for six different values of $\phi_{\max }$. In the calculations, it is assumed that $\phi_{\min }=-\phi_{\max }$. The mode profiles and the color scale are symmetric.

element method. Close inspection of the data indicates that a first-order curvature contribution to the relative frequency shifts vanishes. This result is in agreement with the DGPT theory as we shall show. For a circular-sector torus defined by $\phi_{\min }=-\pi-\phi_{\max }$ with $\phi_{\max }$ in the range $[-\pi / 2$; $\pi / 2]$, the first-order matrix element can be written as

$$
\left\langle l m n\left|\frac{1}{R_{c}} \frac{\partial}{\partial u_{2}}\right| l m n\right\rangle=\frac{1}{R_{c}} \int_{0}^{R} d u_{3} \int_{-h\left(u_{3}\right)}^{h\left(u_{3}\right)} \psi_{l m n}^{0} \frac{\partial \psi_{l m n}^{0}}{\partial u_{2}} d u_{2},
$$

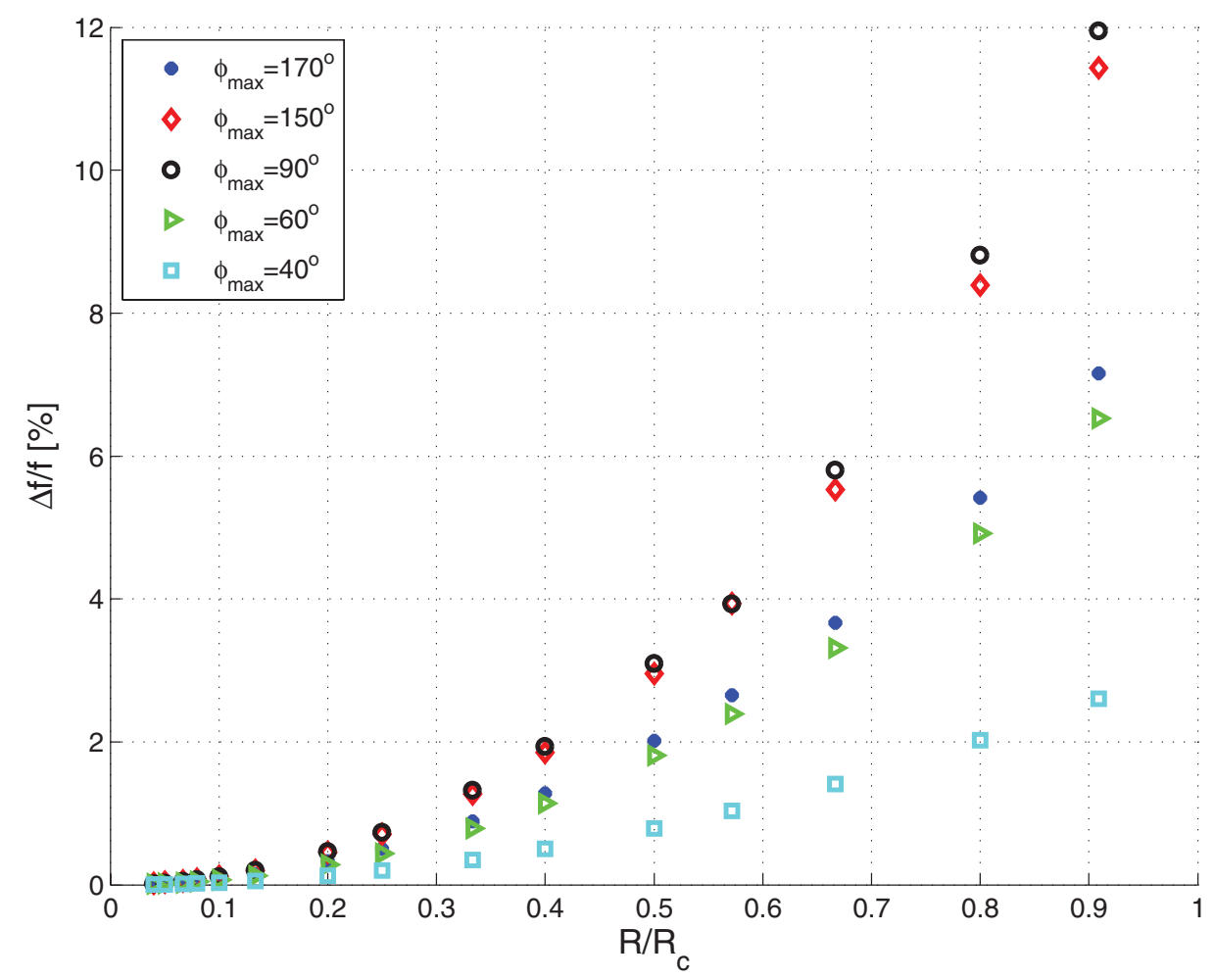

FIG. 8. First-order contribution to $\frac{\Delta f_{l m n}^{N}}{f_{l m n, 0}^{N}}$ as a function of the ratio $R / R_{c}$ corresponding to the mode $p=1, l=0$, $n=1$ for different angles $\phi_{\max }$. Discrete symbol points are found using the FEM. In the calculations, it is assumed that the circular-sector torus spans the angular interval: $\phi_{\min }=-\pi-\phi_{\max }$. 


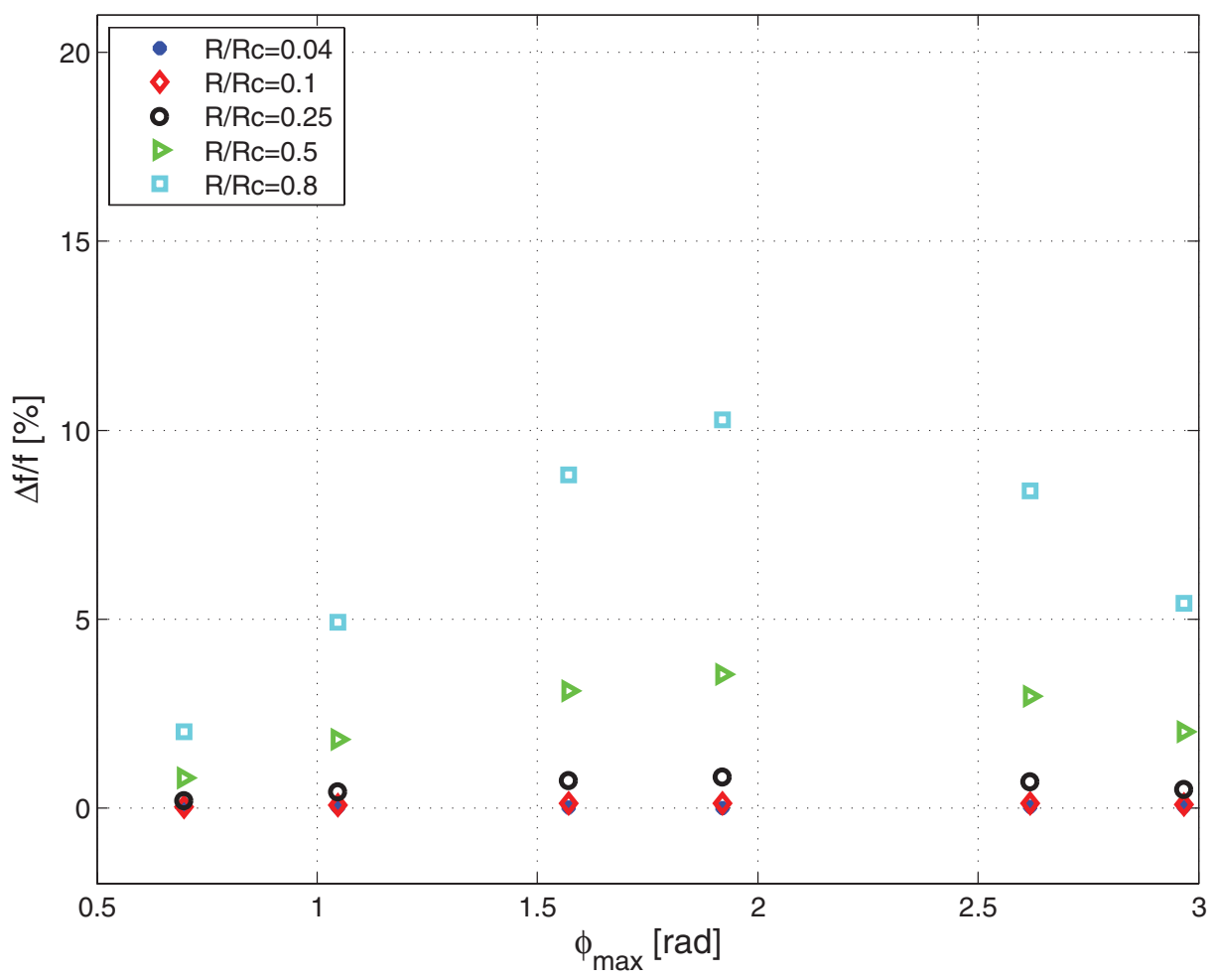

FIG. 9. First-order contribution to $\frac{\Delta f_{l n}^{N}}{f_{l m n, 0}^{N}}$ as a function of $\phi_{\max }$ corresponding to the mode $p=1, l=0, n=1$ for different ratios $R / R_{c}$. Discrete symbol points are found using the FEM. In the calculations, it is assumed that the circular-sector torus spans the angular interval: $\phi_{\min }=-\pi-\phi_{\max }$.

where $h\left(u_{3}\right)$ denotes a function of $u_{3}$ alone. Since $\psi_{\operatorname{lmn}}^{0}$ is a parity eigenstate with eigenvalue $(-1)^{p}$ and $\frac{\partial}{\partial u_{2}} \rightarrow-\frac{\partial}{\partial u_{2}}$ subject to the transformation: $u_{2} \rightarrow-u_{2}$, the latter integral vanishes and the first-order curvature contribution is zero identically. In this argument, we explicitly used that the transformation: $u_{2} \rightarrow-u_{2}$ is a symmetry for circular-sector tori with $\phi_{\min }=-\pi-\phi_{\max }$ but not so for the circular-sector tori discussed earlier defined by $\phi_{\min }=-\phi_{\max }$. However, second-order curvature (and higher order curvature terms) generally contribute in agreement with the FEM results. In Figure 9, we plot discrete data points calculated for the relative frequency shifts as a function of $\phi_{\max }$ for different $R / R_{c}$ ratios using the finite element method. Note that the tendency observed in Figure 6 is recaptured but now for the fundamental mode $p=1, l=0, n=1$, i.e., the relative frequency shifts have a maximum as a function of $\phi_{\max }$ near 1.9 radians equivalent to $109^{\circ}$.

\section{CONCLUSION}

First-order eigenvalue contributions in the curvature for the wave equation problem with Neumann boundary conditions are presented and derived analytically. In the literature hitherto, only second-order in curvature contributions have been identified for Dirichlet and Neumann contributions. This new result has significant implications for, e.g., sound propagation in curved structures and, in particular, the eigenfrequency spectrum. Our analytical results based on differential geometry and perturbation theory are verified by three-dimensional finite element method results applied to a circular-sector torus. It is shown that the corresponding wave equation problem with Dirichlet boundary conditions leads to second-order curvature eigenfrequency contributions only, i.e., a reduced curvature sensitivity for moderately curved waveguides. 
${ }^{1}$ F. H. Borgnis and C. H. Papas, Encyclopedia of Physics: Electromagnetic Waveguides and Resonators (Springer-Verlag, 1958), Vol. 16.

${ }^{2}$ P. M. Morse and H. Feshbach, Methods of Theoretical Physics, Parts I and II (McGraw-Hill, 1953).

${ }^{3}$ P. M. Morse and K. U. Ingard, Theoretical Acoustics (Princeton University Press, 1986).

${ }^{4}$ P. Moon and D. E. Spencer, Field Theory Handbook (Springer-Verlag, Berlin, 1961).

${ }^{5}$ J. D. Jackson, Classical Electrodynamics, 3rd ed. (Wiley, 1999).

${ }^{6}$ M. Willatzen and L. C. Lew Yan Voon, Separable Boundary-Value Problems in Physics (Wiley-VCH, 2011).

${ }^{7}$ H. Jensen and H. Koppe, Ann. Phys. (Leipzig) 63, 586 (1971).

${ }^{8}$ R. C. T. da Costa, Phys. Rev. 23, 1982 (1981).

${ }^{9}$ R. C. T. da Costa, Phys. Rev. A 25, 2893 (1982).

${ }^{10}$ J. Goldstone and R. L. Jaffe, Phys. Rev. B 45, 14100 (1992).

${ }^{11}$ P. Exner and P. Seba, J. Math. Phys. 30, 2574 (1989).

${ }^{12}$ N. E. Hurt, Mathematical Physics of Quantum Wires and Devices, Spectral Resonances to Anderson Localization Series: Mathematics and Its Applications Vol. 506 (Springer, 2000).

${ }^{13}$ S. Tanda, T. Tsuneta, T. Toshima, T. Matsuura, and M. Tsubota, J. Phys. IV 131, 289 (2005).

${ }^{14}$ M. König, S. Wiedmann, C. Brüne, A. Roth, H. Buhmann, L. W. Molenkamp, X.-L. Qi, and S.-C. Zhang, Science 318, 766 (2007).

${ }^{15}$ Y. Ran, Y. Zhang, and A. Vishwanath, Nat. Phys. 5, 298 (2009).

${ }^{16}$ J. Gravesen, M. Willatzen, and L. C. Lew Yan Voon, J. Math. Phys. 46, 012107 (2005)

${ }^{17}$ J. Gravesen and M. Willatzen, Physica B 371, 112 (2006).

${ }^{18}$ J. Gravesen and M. Willatzen, Phys. Rev. A 72, 032108 (2005).

${ }^{19}$ J. Gravesen, M. Willatzen, and L. C. Lew Yan Voon, Phys. Scr. 72, 105 (2005).

${ }^{20}$ E. L. Starostin and G. H. M. van der Heijden, Phys. Rev. Lett. 101, 084301 (2008).

${ }^{21}$ M. Encinosa and B. Eternadi, Phys. Rev. A 58, 77 (1998).

${ }^{22}$ P. W. Anderson, Phys. Rev. 109, 1492 (1958).

${ }^{23}$ J. D. Joannopoulos, R. D. Meade, and J. N. Winn, Photonic Crystals: Molding the Flow of Light (Princeton University, 1995).

${ }^{24}$ M. M. Sigalas and E. N. Economou, Solid State Commun. 86, 141 (1993).

${ }^{25}$ M. S. Kushwaha, Int. J. Mod. Phys. B 10, 977 (1996).

${ }^{26}$ A. A. Krokhin, J. Arriaga, and L. N. Gumen, Phys. Rev. Lett. 91, 264302 (2003).

${ }^{27}$ M. Willatzen, J. Gravesen, and L. C. Lew Yan Voon, Phys. Rev. A 81, 060102(R) (2010). 